\title{
On the tonehole lattice cutoff frequency of conical resonators: applications to the saxophone
}

\author{
Erik Petersen*, Tom Colinot, Jean Kergomard, and Philippe Guillemain \\ LMA, CNRS, UPR 7051, Aix-Marseille Univ, Centrale Marseille, 13453 Marseille Cedex 13, France
}

Received 17 January 2020, Accepted 20 July 2020

\begin{abstract}
The tonehole lattice cutoff frequency is a well-known feature of woodwind instruments. However, most analytic studies of the cutoff have focused on cylindrical instruments due to their relative geometric simplicity. Here, the tonehole lattice cutoff frequency of conical instruments such as the saxophone is studied analytically, using a generalization of the framework developed for cylindrical resonators. First, a definition of local cutoff of a conical tonehole lattice is derived and used to design "acoustically regular" resonators with determinate cutoff frequencies. The study is then expanded to an acoustically irregular lattice: a saxophone resonator, of known input impedance and geometry. Because the lattices of real instruments are acoustically irregular, different methods of analysis are developed. These methods, derived from either acoustic (input impedance) or geometric (tonehole geometry) measurements, are used to determine the tonehole lattice cutoff frequency of conical resonators. Each method provides a slightly different estimation of the tonehole lattice cutoff for each fingering, and the range of cutoffs across the first register is interpreted as the acoustic irregularity of the lattice. It is shown that, in contrast with many other woodwind instruments, the cutoff frequency of a saxophone decreases significantly from the high to low notes of the first register.
\end{abstract}

Keywords: Woodwinds, Tonehole lattice, Saxophone, Cutoff frequency

\section{Introduction}

Woodwind instruments such as the clarinet and saxophone have resonators that are composed of an acoustical duct for which the downstream section has a lattice of toneholes that can be opened and closed to change the playing frequency of the instrument. At low frequencies, the effective acoustical length of the resonator is defined by the distance between the mouthpiece and the first open tonehole because an acoustical wave, upon arrival at the tonehole, either radiates into the surrounding environment or reflects back into the main bore of the instrument. At higher frequencies, the wave is no longer evanescent and propagates into the remaining lattice of toneholes. For a real instrument of finite length, the (approximate) tonehole lattice cutoff frequency separates these two bands, although no definition is universally agreed upon and a precise definition does not exist because the toneholes do not constitute a perfectly periodic lattice.

The tonehole lattice cutoff frequency has been studied for various instruments, particularly the clarinet, but relatively little application to the saxophone exists in the literature $[1,2]$. In fact, in his book, Benade provides the input impedance and discusses (approximate) cutoff frequencies

*Corresponding author: erikalanpetersen@gmail.com for many instruments, including the clarinet, oboe, bassoon and tárogató, but not the saxophone [3]. The cutoff has also been studied for the flute [4]. It is generally assumed that the cutoff frequency has an impact on the timbre or perceived "character" of a given instrument due to its influence on both sound production and radiation, and is therefore of interest to both instrument makers and musicians [3, 5].

A precise definition of the tonehole lattice cutoff frequency does not exist due to the finite and lossy nature of real instruments [6]. Therefore, only approximate definitions exist, which may be either empirical or analytic. A summary of the notation used in the current article is provided in Table 1. One way to define the cutoff frequency is to identify a disturbance in the measured or simulated input impedance or reflection coefficient, and set an arbitrary threshold to distinguish between the pass and stop bands. Often the disturbance is identified visually from the input impedance, which introduces questions about reproducibility, and the uncertainty can be on the order of the distance between impedance peaks. Regardless, this is the most common method for identifying the cutoff of woodwind instruments. A second empirical method to identify the cutoff frequency uses measurements external to the instrument during playing conditions across a large range of the instrument. From this, Benade defines a "break frequency," and related it to the cutoff frequency, which is at the 
Table 1. Summary of the different types of cutoff frequencies used in the current article.

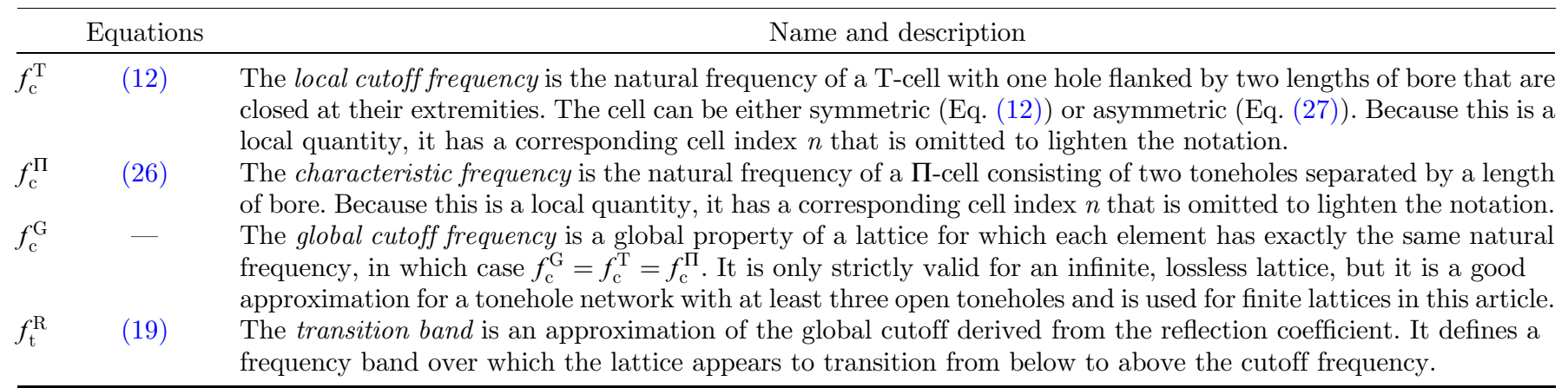

A lattice is geometrically regular if every cell is geometrically identical. In this case every cell has the same $f_{\mathrm{c}}^{\mathrm{T}}$ (and $f_{\mathrm{c}}^{\Pi}$ ) and the lattice has a global cutoff $f_{\mathrm{c}}^{\mathrm{G}}$. This is not possible for a conical lattice because no two cells can be identical due to the taper of the bore.

A lattice is acoustically regular if every cell has the same local cutoff $f_{\mathrm{c}}^{\mathrm{T}}$ (and $f_{\mathrm{c}}^{\Pi}$ ), resulting in a global cutoff $f_{\mathrm{c}}^{\mathrm{G}}$. Conical bores can be acoustically regular because the cross-section and chimney height of a tonehole can be modified to account for the changing crosssection of the main bore.

intersection of the low frequency rise and high frequency roll-off of the spectrum envelope [7]. Both of these methods are prone to ambiguous results for certain fingerings and instruments. While empirically estimated cutoff frequencies are often assumed to be due to the tonehole lattice, it is not always a robust method because other components, such as the bell, can cause similar disturbances in the input impedance.

Analytically, the global cutoff frequency $f_{\mathrm{c}}^{\mathrm{G}}$ can be developed following the theory of wave propagation in periodic media [6], in which a cutoff frequency can be precisely defined for an infinite, lossless lattice. Here, local quantities corresponding to the unit cells of the lattice define the global cutoff frequency. In the case where the medium is a column of air punctuated by toneholes, the lattice is divided into discrete elements in the form of T-cells, a hole with flanking lengths of duct on either side, or as П-cells with two neighboring (partial) holes and the length of duct between them. For either shape, an eigenfrequency can be calculated for each cell in the lattice, referred to as the local cutoff frequency $f_{\mathrm{c}}^{\mathrm{T}}$ and characteristic frequency $f_{\mathrm{c}}^{\Pi}$, respectively. If each cell in the lattice has the same eigenfrequency, then the lattice is considered an acoustically regular (periodic) medium, and the lattice formally has a global cutoff frequency that is equal to the common eigenfrequency of each constituent cell. Periodic cylindrical lattices are easily designed by concatenating identical unit cells to form geometrically regular lattices. For conical resonators, which cannot be geometrically regular due to the taper of the main bore's internal radius, a lattice can be periodic following the interpretation of acoustical periodicity. While these derivations are only strictly valid for an infinite, lossless lattice, the theory works well for lattices with as few as three toneholes.

The purpose of the current article is to generalize the theory of wave propagation in periodic media from cylindrical to conical resonators. In a recent article, the current authors present an algorithm for designing cylindrical resonators with independently variable first impedance peak and global cutoff frequencies [5]. Analogous equations are derived for conical resonators in Section 2. Section 3 applies the analysis of a conical tonehole lattice to the geometry of a Buffet Crampon alto saxophone. This section also includes a comparison of the various cutoff frequency definitions and approximations as they apply to simplified resonators and the Buffet Crampon alto saxophone. Conclusions and perspectives are provided in Section 4.

\section{Acoustically regular conical tonehole lattice}

In this section a conical resonator is designed such that the frequency $f_{1}$ of the first impedance peak and the global tonehole lattice cutoff frequency $f_{c}^{\mathrm{G}}$ can be independently varied. Inspired by the geometry of a saxophone resonator without a mouthpiece, this is achieved by using a length of cone that ends with a series of acoustically regular lattice cells. The input impedance peaks below the global cutoff frequency have relatively large magnitudes that are approximately harmonically related to the first peak. Above the global cutoff frequency the peaks and troughs are less pronounced and may not have the same organized spacing in frequency. This is also seen in the reflection coefficient, which has a value close to unity below the global cutoff frequency, and drops significantly at the cutoff. An example of a synthesized input impedance and reflection coefficient is shown in Figure 1 and discussed in greater detail in Section 2.4.

In the following sections we derive the equations that describe the conditions for acoustic regularity of a conical waveguide. The derivation uses T-cells as the basic element of the lattice, although it is also possible to start with П-cells.

\subsection{Transfer matrix equations of a conical lattice}

\subsubsection{Basic equations}

In the following derivation, a conical lattice is designed by concatenating $n$ cells that have a tonehole at the center 

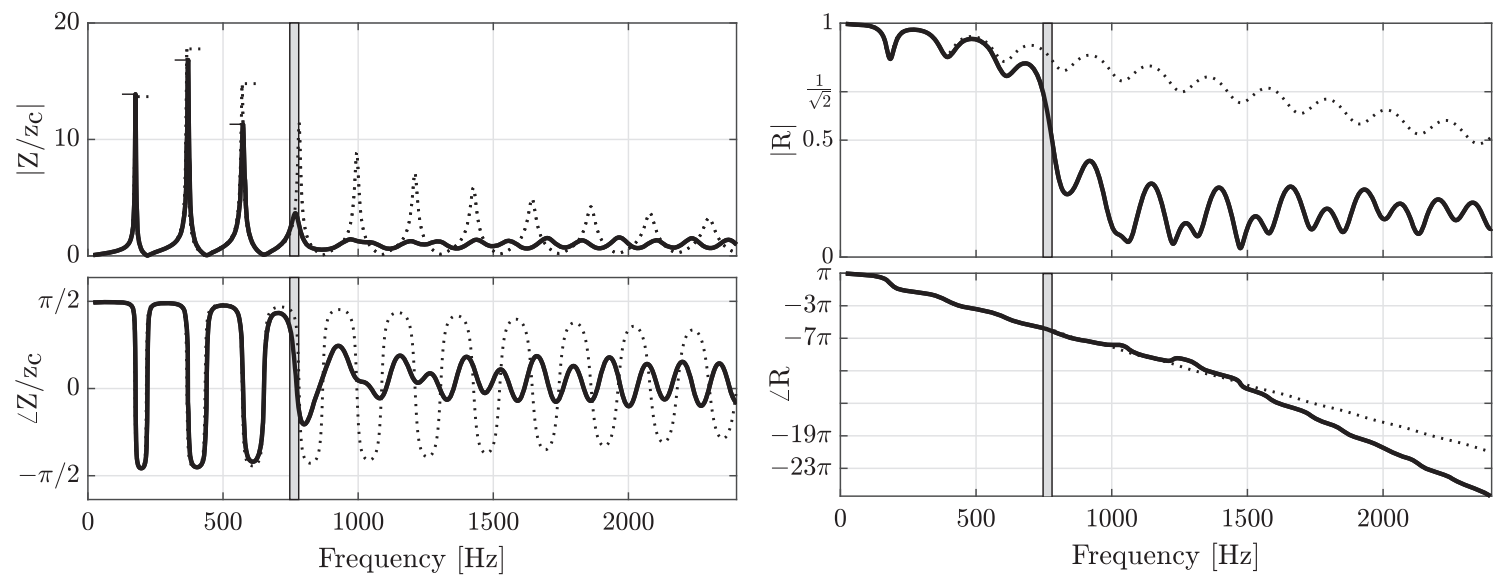

Figure 1. Input impedance (modulus and argument) and reflection coefficient (modulus and argument) of an acoustically regular conical resonator (solid black lines) with a first impedance peak at $177 \mathrm{~Hz}$ and a theoretical global tonehole lattice cutoff frequency at $750 \mathrm{~Hz}$. A conical resonator (dotted lines) with the same first impedance peak frequency but no tonehole lattice is shown for comparison. The first three impedance modulus peaks are marked by a solid black line (resonator with a lattice) and dotted lines (simple cone). The cutoff transition band $f_{\mathrm{t}}^{\mathrm{R}}$ (defined in Sect. 2.4, Eq. (19)) is shown in shaded grey.
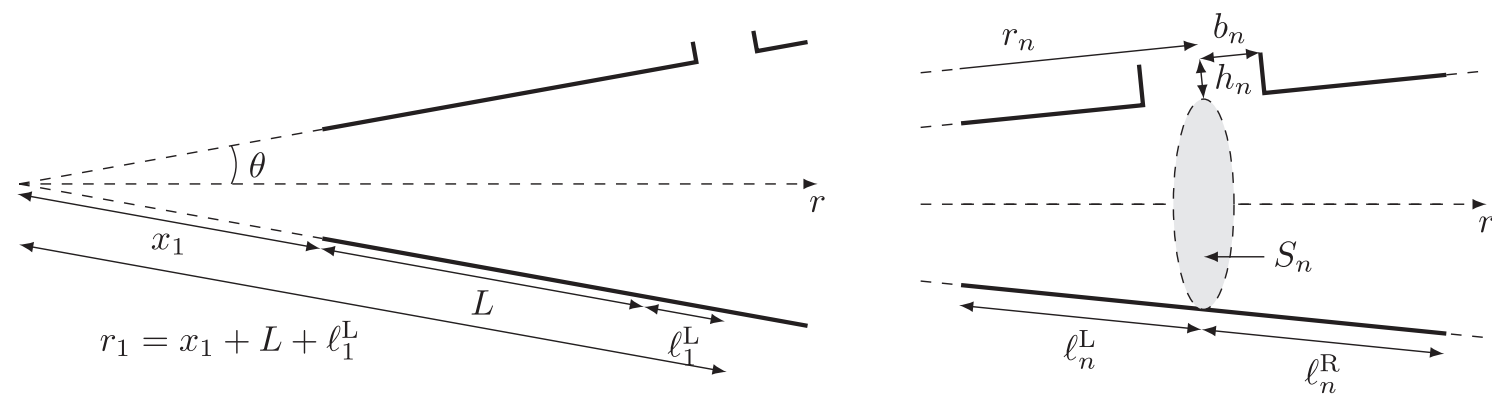

Figure 2. Schematic of a conical resonator with apex angle $\theta$ and a lattice of toneholes. There is a length of duct $L$ before the first symmetric T-cell centered at $r_{1}$ (left panel). The missing length of the cone is $x_{1}$. The $n$th T-cell, centered at $r_{n}$, is flanked by cones of length $\ell_{n}^{\mathrm{L}}$ and $\ell_{n}^{\mathrm{R}}$ on either side (right panel). The tonehole has cross-section $s_{n}=\pi b_{n}^{2}$ and chimney height $h_{n}$. The cross-section of the main bore under the tonehole is $S_{n}$.

and truncated cones of length $\ell_{n}^{\mathrm{L}}$ and $\ell_{n}^{\mathrm{R}}$ to the left and right, as shown in Figure 2. The frequency domain Helmholtz equation for the acoustic pressure $P(r, k)$ is,

$$
\frac{\partial^{2}}{\partial r^{2}}[r P(r, k)]+k^{2} r P(r, k)=0
$$

where $k=\omega / c$ is the wavenumber with $\omega$ the angular frequency and $c$ the speed of sound in air. Thermoviscous and radiation losses are ignored. The acoustic velocity $V$ $(r, k)$ is derived from the Euler equation, $\partial_{r} P(r, k)=$ $-j \rho c k V(r, k)$, where $\rho$ is the density of air and $j$ the imaginary unit.

A small angle approximation of the apex angle $\theta$ is applied for the following derivation. The approximation assumes that the difference between the spherical cap of a propagating wave and a flat surface cross-section of the main bore can be ignored. Similarly, the difference between a length along the axis $r$ and its projection on the edge of the cone is ignored. For a typical apex angle $\theta=0.028 \mathrm{rad}$ these approximations introduce less than $0.1 \%$ error for the surface and less than $0.5 \%$ error for the lengths. The advantage of this approximation is that it assumes planar waves, such that the pressure is constant across a flat surface cross-section of the main bore, allowing the use of a simple tonehole model.

\subsubsection{Transfer matrix across a single cell}

The relation between pressure and velocity across a section of the waveguide is written as a transfer matrix equation,

$$
\left(\begin{array}{c}
r_{1} P\left(r_{1}, k\right) \\
r_{1} V\left(r_{1}, k\right)-\frac{P\left(r_{1}, k\right)}{j c k \rho}
\end{array}\right)=M_{1,2}(k)\left(\begin{array}{c}
r_{2} P\left(r_{2}, k\right) \\
r_{2} V\left(r_{2}, k\right)-\frac{P\left(r_{2}, k\right)}{j c k \rho}
\end{array}\right),
$$

where the matrix $M_{1,2}(k)$ (written generically here, and with superscript labels and subscript indices when referring to specific sections) has elements $A(k), B(k), C(k)$, and $D(k)$ that correspond to the geometry of the waveguide sections. The transfer matrix relating pressure and velocity across the $n$th cell (see Fig. 2, right panel) centered at $r_{n}$ is the product of the transfer matrices across 
the tonehole $M_{n}^{\mathrm{h}}(k)$ and the left and right flanking conical sections $M_{n}^{\mathrm{L}}(k)$ and $M_{n}^{\mathrm{R}}(k)$,

$$
M_{n}(k)=M_{n}^{\mathrm{L}}(k) M_{n}^{\mathrm{h}}(k) M_{n}^{\mathrm{R}}(k) .
$$

It is convenient, although not necessary, to consider "symmetric" cells (truly symmetric cells cannot exist due to the taper of the main bore) for which $\ell_{n}^{\mathrm{L}}=\ell_{n}^{\mathrm{R}}=\ell_{n}$,

$$
M_{n}(k)=M_{n}^{\ell}(k) M_{n}^{\mathrm{h}}(k) M_{n}^{\ell}(k) .
$$

The remainder of this derivation assumes symmetric cells, and asymmetric cells are treated in Section 3.

The transfer across a truncated cone of length $\ell_{n}$ is,

$$
M_{n}^{\ell}(k)=\left(\begin{array}{cc}
\cos \left(k \ell_{n}\right) & j \rho c \sin \left(k \ell_{n}\right) \\
\frac{j}{\rho c} \sin \left(k \ell_{n}\right) & \cos \left(k \ell_{n}\right)
\end{array}\right) .
$$

A tonehole is treated as a shunt acoustic mass: $m_{n}=\rho h_{n} / s_{n}$, where $s_{n}=\pi b_{n}^{2}$ is the cross-sectional area of the tonehole and the chimney height $h_{n}$ includes the internal and radiation length corrections. The transfer matrix across the $n$th tonehole is,

$$
M_{n}^{\mathrm{h}}(k)=\left(\begin{array}{cc}
1 & 0 \\
Y_{n}(k) & 1
\end{array}\right),
$$

where $Y_{n}(k)=1 /\left(j c k m_{n} S_{n}\right)$ is the specific admittance of the tonehole.

The transfer matrix equation relating $P(r, k)$ and $V(r, k)$ from $r_{n}-\ell_{n}$ to $r_{n}+\ell_{n}$ across the entire $n$th symmetric cell centered at $r_{n}$ is,

$$
\begin{aligned}
& \left(\begin{array}{c}
\left(r_{n}-\ell_{n}\right) P\left(r_{n}-\ell_{n}, k\right) \\
\left(r_{n}-\ell_{n}\right) V\left(r_{n}-\ell_{n}, k\right)-\frac{P\left(r_{n}-\ell_{n}, k\right)}{j c k \rho}
\end{array}\right)= \\
& M_{n}(k)\left(\begin{array}{c}
\left(r_{n}+\ell_{n}\right) P\left(r_{n}+\ell_{n}, k\right) \\
\left(r_{n}+\ell_{n}\right) V\left(r_{n}+\ell_{n}, k\right)-\frac{P\left(r_{n}+\ell_{n}, k\right)}{j c k \rho}
\end{array}\right)
\end{aligned}
$$

where $M_{n}(k)$ is determined by equation (4) using equations (5) and (6), has elements classically denoted $A_{n}(k)$, $B_{n}(k), C_{n}(k), D_{n}(k)$ and its determinant is unity due to reciprocity.

\subsubsection{From one cell to full lattice}

The transfer matrix $M$ for a lattice with $N$ cells is the matrix product of the transfer matrix of each cell,

$$
M(k)=\prod_{n=1}^{N} M_{n}(k)=M_{1}(k) M_{2}(k) \cdots M_{N}(k) .
$$

The resulting matrix,

$$
M(k)=\left(\begin{array}{ll}
A(k) & B(k) \\
C(k) & D(k)
\end{array}\right),
$$

has coefficients $A_{n}(k), B_{n}(k), C_{n}(k), D_{n}(k)$ that can be used to calculate global features of the lattice such as the input impedance.
So far, the lattice modeled by $M(k)$ is not necessarily acoustically regular and may not have a global cutoff frequency. To create a lattice that is acoustically regular and exhibits a global cutoff it is necessary to impose conditions on the local elements accounted for by each cell $M_{n}(k)$.

\subsection{The cutoff frequency of an acoustically regular lattice}

For the pressure $P(r, k)$ defined at the input $r=r_{n}-\ell_{n}$ of the $n$th cell, an acoustic periodicity implies,

$$
\begin{array}{r}
2 A_{n}(k)\left(r_{n}-\ell_{n}\right) P\left(r_{n}-\ell_{n}, k\right)=\left(r_{n-1}-\ell_{n-1}\right) P\left(r_{n-1}-\ell_{n-1}, k\right) \\
+\left(r_{n+1}-\ell_{n+1}\right) P\left(r_{n+1}-\ell_{n+1}, k\right),
\end{array}
$$

which is derived from equation (7). An explicit expression for $A_{n}(k)$ can be determined from the geometry of each cell, and the frequency $f=c k /(2 \pi)$ that satisfies $A_{n}(k)=D_{n}(k)= \pm 1$ for a given cell is the local cutoff frequency $f_{\mathrm{c}}^{\mathrm{T}}$. When $A_{n}(k)= \pm 1$ occurs at the same frequency for every cell, the lattice is acoustically regular and has a global cutoff frequency $f_{\mathrm{c}}^{\mathrm{G}}$, below which waves entering the lattice are evanescent [8].

The transfer matrix elements $A_{n}(k)$ and $D_{n}(k)$ that define the local cutoff of a cell can be calculated directly from the geometry of the $n$th cell. Therefore, it is possible to choose the cell geometry to achieve a desired local cutoff frequency. For a symmetric cell defined by equations (4)-(6) and setting $A_{n}(k)=1$, a transcendental equation defines the local cutoff in terms of cell geometry,

$$
\cot \left(k_{\mathrm{c}}^{\mathrm{T}} \ell_{n}\right)=2 k_{\mathrm{c}}^{\mathrm{T}} h_{n} S_{n} / s_{n},
$$

where $k_{\mathrm{c}}^{\mathrm{T}}=2 \pi f_{\mathrm{c}}^{\mathrm{T}} / c$. The local cutoff frequency is approximated using a Taylor expansion of the cotangent in $k_{\mathrm{c}}^{\mathrm{T}} \ell_{n}$,

$$
\begin{gathered}
f_{\mathrm{c}}^{\mathrm{T}}=\frac{c}{2 \pi \ell_{n}}\left[\frac{1}{\sqrt{2\left(h_{n} / \ell_{n}\right)\left(S_{n} / s_{n}\right)+1 / 3}}+\mathcal{O}\left(\left(k_{\mathrm{c}}^{\mathrm{T}} \ell_{n}\right)^{3}\right)\right], \\
f_{\mathrm{c}}^{\mathrm{T}}=\frac{c}{2 \pi \ell_{n}}\left[\frac{1}{\sqrt{2\left(h_{n} / \ell_{n}\right)\left(S_{n} / s_{n}\right)}}+\mathcal{O}\left(k_{\mathrm{c}}^{\mathrm{T}} \ell_{n}\right)\right],
\end{gathered}
$$

where the approximations correspond to expansions retaining two and one terms, respectively. Both of these expansions of the cotangent function are valid assuming $\left(k_{\mathrm{c}}^{\mathrm{T}} \ell_{n}\right)^{2} \ll 1$, for which the half-spacing between holes is much smaller than the wavelength at the local cutoff, and the cell can be treated as a lumped element system. For a local cutoff at $900 \mathrm{~Hz}$ and a common interhole spacing $2 \ell_{n} \approx 0.04 \mathrm{~m},\left(k_{\mathrm{c}}^{\mathrm{T}} \ell_{n}\right)^{2} \approx 0.1$ and the condition is satisfied. As for the case of a cylindrical cell [1], the local cutoff frequency can be interpreted as the eigenfrequency of a single cell with Neumann boundary conditions satisfied at the left and right extremities of the main bore.

One way to quantify the tonehole lattice global cutoff frequency of an acoustically regular lattice is to examine the acoustic flow $U(\omega)$ through each radiating aperture, relative to the first tonehole. Figure 3 shows the transfer function $H_{n}^{U}(\omega)=20 \log _{10}\left(U_{n}(\omega) / U_{1}(\omega)\right)$ for the 


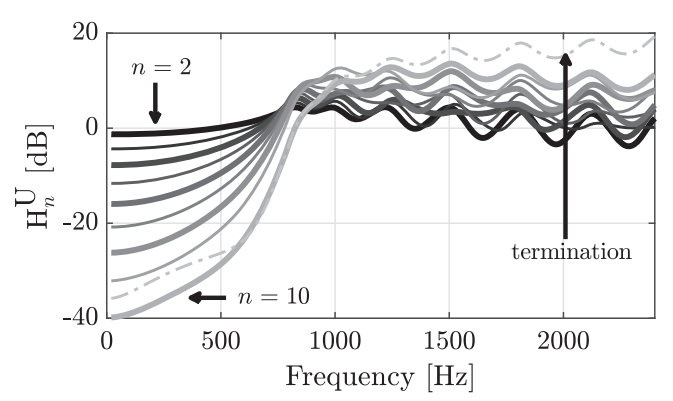

Figure 3. The transfer function between flow through the first tonehole and flow through the $n$th (for $n=2-10$ ) tonehole and open termination of a conical lattice with $f_{\mathrm{c}}^{\mathrm{G}}=750 \mathrm{~Hz}$. The lattice geometry is Resonator 1 in the Appendix, and is the same lattice used for Figure 1.

acoustically regular lattice with geometry provided in the Appendix, also depicted in Figure 1. These simulations follow the method outlined in Section 2.4. Below global cutoff, the flow is predominantly through the first tonehole, with an exponential decay of approximately $4.8 \mathrm{~dB}$ (at $50 \mathrm{~Hz}$ ) for each subsequent tonehole. Above the global cutoff, the flow is predominantly through the termination of the lattice, with considerable contributions from multiple toneholes. This figure is the conical lattice counterpart to the results for measured $[9,10]$ and analytically studied cylindrical lattices [11].

\subsection{From lattice to a saxophone-type resonator}

To better mimic the acoustical behavior of a real alto saxophone, the resonator is designed as a conical bore approximately $60 \mathrm{~cm}$ long that terminates in an acoustically regular lattice designed following the method proposed in Section 2.2 using equation (12). Dimensions of two possible examples are provided in the Appendix. The upstream (closest to the mouthpiece) section of the cone determines the low frequency resonances of the resonator, and the lattice of open toneholes modifies the high frequency (above the global cutoff) resonances by changing the effective acoustical length. It is possible to design a resonator such that the frequency of the first impedance peak and the global cutoff frequency of the lossless tonehole lattice can be varied independently [5].

The length of a lattice-less frustum (cone truncated on both ends) with a desired first impedance resonance frequency $f_{1}$ is,

$$
L_{\text {eff }}\left(\lambda_{1}, x_{1}\right)=\lambda_{1}\left(\frac{1}{2}-\frac{1}{2 \pi} \arctan \left(\frac{2 \pi x_{1}}{\lambda_{1}}\right)\right),
$$

where $\lambda_{1}=c / f_{1}$ is the wavelength corresponding to the first impedance peak frequency and $x_{1}$ the missing length between the apex and small end of the frustum. At low frequencies, a lattice of toneholes changes the terminal impedance of the cone by adding a mass-like term that can be expressed as a length correction $\Delta \ell_{1}$ [8]. This length correction is a function of the first cell length $\ell_{1}^{\mathrm{L}}$ and the wavelength $\lambda_{c}$ at the local cutoff,

$$
\Delta \ell_{1}\left(\lambda_{c}, \ell_{1}^{\mathrm{L}}\right)=\ell_{1}^{\mathrm{L}} \sqrt{1+\left(\frac{\lambda_{\mathrm{c}}}{2 \pi \ell_{1}^{\mathrm{L}}}\right)^{2}} .
$$

The end correction term implies that a conical resonator without a lattice (but same $f_{1}$ ) should be slightly longer than the length between the input and first hole of a resonator with a lattice. Therefore, the segment of the resonator before the lattice should have a length,

$$
L=L_{\text {eff }}-\ell_{1}^{\mathrm{L}}-\Delta \ell_{1},
$$

depicted in the left panel of Figure 2.

These equations provide the basic algorithm for designing a resonator such that the first resonance frequency and the global tonehole lattice cutoff can be independently varied. It is possible to conceive different geometries that result in an acoustically regular tonehole lattice with the same global cutoff. In the current work, the geometries are inspired by, but not identical to, a Buffet Crampon alto saxophone.

\subsection{Simulated impedance of acoustically regular lattice and equivalent truncated cone}

The input impedance is simulated to demonstrate the validity of an acoustically regular conical lattice design. The simulations are based on the Transfer Matrix Method with external Interactions (TMMI), which is similar to the well-known Transfer Matrix Method, but includes the mutual impedance of toneholes that radiate into the same space [12]. In contrast with the previous theoretical development, the TMMI simulations include thermoviscous and radiation losses. The resonator has a first impedance peak at $177 \mathrm{~Hz}$ and a global cutoff at $750 \mathrm{~Hz}$. The geometry of this academic resonator and another with a global cutoff at $1000 \mathrm{~Hz}$ is provided in the Appendix.

Figure 1 shows the simulated input impedance,

$$
Z(\omega)=P(\omega) / U(\omega),
$$

normalized by $\mathrm{z}_{\mathrm{c}}=\rho c /\left(\pi a_{\text {in }}^{2}\right)$ where $a_{\text {in }}=x_{1} \tan (\theta)$ is the radius of the entrance to the bore, and the corresponding reflection coefficient,

$$
R(\omega)=\frac{Z(\omega) / \mathrm{z}_{\mathrm{c}}-1}{Z(\omega) / \mathrm{z}_{\mathrm{c}}+1} .
$$

The effect of the global cutoff frequency can be identified visually in the magnitude and argument of both the input impedance and reflection coefficient. The large and regularly spaced impedance peaks whose frequencies are determined by the upstream portion of the resonator are obliterated above the global cutoff because the wave propagates into the lattice and reflections back into the bore are weak. Furthermore, above the global cutoff the wave radiates from subsequent toneholes and experiences greater thermoviscous losses due to the increased propagation distance within the bore. These combined effects result in the disturbance seen in the input impedance and reflection coefficient.

Because the lattice is designed to have acoustically regular cells, the local (and therefore global) cutoff frequencies 
are, in principle, exactly $750 \mathrm{~Hz}$. As noted before, it is not always sensible to define a precise, unambiguous definition of the cutoff from the input impedance and reflection coefficient. Therefore, a transition band $f_{\mathrm{t}}^{\mathrm{R}}$ shown in light grey is defined by,

$$
f_{\min } \leq f_{\mathrm{t}}^{\mathrm{R}} \leq f_{\max },
$$

where the lower and upper bounds $f_{\min }$ and $f_{\max }$ correspond to the -3 and $-6 \mathrm{~dB}$ roll off of the reflection coefficient. This is determined by the lowest frequencies that satisfy,

$$
\begin{aligned}
& \left|R\left(2 \pi f_{\min }\right)\right|=\frac{\sqrt{2}}{2}, \\
& \left|R\left(2 \pi f_{\max }\right)\right|=\frac{1}{2} .
\end{aligned}
$$

Although the thresholds are arbitrary, this transition band serves as an objective empirical estimation of the global cutoff frequency for the remainder of this article. Note that it is distinct from a theoretically defined (global, local, or characteristic) cutoff, summarized in Table 1.

The dashed curve in Figure 1 shows the input impedance of a cone which terminates in a radiation load but no lattice. Its length is chosen so that the first impedance peak frequency is the same as that of the acoustically regular resonator that terminates with a lattice. This serves as a comparison to show which features of the input impedance are due to the tonehole lattice, and which arise solely from the conical geometry. As for the resonator with a tonehole lattice, the reflection coefficient of the simple cone also has regularly spaced dips. This behavior is due to the conical geometry of the waveguide, and is therefore seen in the simulations of both resonators, even below the gobal cutoff frequency of the resonator with a tonehole lattice.

For the readability of Figure 1, the cutoff transition band $f_{\mathrm{t}}^{\mathrm{R}}$ is not shown for the lattice-less cone. However, if the definition was applied, the threshold conditions are met for a range approximately $1250-2300 \mathrm{~Hz}$. For this reason, the tonehole lattice cutoff frequency of a conical resonator is difficult to identify empirically in part because there are multiple phenomena that lead to similar measured behavior: the tonehole lattice, the conicity of the main bore, and the bell (not treated in the current article), can all cause perturbations of the input impedance and dips in the reflection coefficient. This may explain why there are comparatively few articles about the cutoff frequency of conical instruments such as the saxophone, and demonstrates the utility of using simplified resonators to study the phenomenon. In particular, the features that are evident in Figure 1 are helpful to guide the discussion for resonators that are not strictly acoustically regular.

\section{Application to the saxophone: acoustically irregular lattices}

Real saxophones do not have strictly acoustically regular tonehole lattices. However, cutoff frequency type behaviors are observed in the input impedance measurements for many fingerings of the saxophone, for which two example fingerings are provided in Figures 4 and 5. In addition, the toneholes tend to increase in radius and interhole spacing progressively along the resonator, which suggests an approximate acoustic regularity of the type defined by equation (12). Therefore, it is still interesting to apply the theory of a tonehole lattice cutoff frequency to the saxophone.

A practical complication in assessing the acoustic regularity of a real instrument is precising the geometry of the tonehole cells because the lattice cannot, in general, be divided into symmetric T-cells. Can the geometry of a real saxophone be divided into cells such that local cutoff frequencies can be determined? This question is explored using $\Pi$-cells and asymmetric T-cells, and the methods are applied to the geometry of a Buffet Crampon alto saxophone [1].

\subsection{Tonehole cell pairs}

The first method to characterize the acoustic regularity of a lattice considers not the local eigenfrequencies of constituent T-cells, but rather defines the characteristic frequency $f_{\mathrm{c}}^{\Pi}$ (Eq. (25) and Table 1) of adjacent toneholes and the length of cone separating the pair, often referred to as a $\Pi$-cell. This way, the characteristic frequency of each tonehole pair in a lattice can be unambiguously calculated directly from the measured geometry of an instrument. As for a lattice of T-cells, if each П-cell in a lattice has the same characteristic frequency then the lattice as a global cutoff $f_{\mathrm{c}}^{\mathrm{G}}=f_{\mathrm{c}}^{\Pi}$.

If the eigenfrequencies of adjacent symmetric T-cells are equal,

$$
\begin{aligned}
& f_{\mathrm{c}}^{\mathrm{T}}=\frac{c}{2 \pi \ell_{n}}\left[\frac{1}{\sqrt{2\left(h_{n} / \ell_{n}\right)\left(S_{n} / s_{n}\right)}}+\mathcal{O}\left(k_{\mathrm{c}}^{\mathrm{T}} \ell_{n}\right)\right] \\
& =\frac{c}{2 \pi \ell_{n+1}}\left[\frac{1}{\sqrt{2\left(\frac{h_{n+1}}{\ell_{n+1}}\right)\left(\frac{S_{n+1}}{s_{n+1}}\right)}}+\mathcal{O}\left(k_{\mathrm{c}}^{\mathrm{T}} \ell_{n+1}\right)\right],
\end{aligned}
$$

then their geometries are related such that,

$$
\ell_{n} h_{n} \frac{S_{n}}{S_{n}}=\ell_{n+1} h_{n+1} \frac{S_{n+1}}{S_{n+1}}=\frac{c^{2}}{2\left(2 \pi f_{\mathrm{c}}^{\mathrm{T}}\right)^{2}} .
$$

As shown in Figure 6, the spacing between the two holes is denoted $d_{n}=\ell_{n}^{\mathrm{R}}+\ell_{n+1}^{\mathrm{L}}$, where the assumption of symmetric T-cells is no longer necessary. Retaining one term of the Taylor expansion of equation (11) yields the characteristic frequency of a tonehole pair,

$$
f_{\mathrm{c}}^{\Pi}=\frac{c}{2 \pi d_{n}}\left[\frac{1}{\sqrt{2\left(K_{n}+K_{n+1}^{\prime}\right)}}+\mathcal{O}\left(k_{\mathrm{c}}^{\mathrm{T}} \ell_{n}\right)\right],
$$

where the dimensionless $K_{n}=\frac{h_{n}}{d_{n}} \frac{S_{n}}{s_{n}}$ and $K_{n+1}^{\prime}=\frac{h_{n+1}}{d_{n}} \frac{S_{n+1}}{s_{n+1}}$ are introduced for brevity. The subscript of $d_{n}$ does not increment to $d_{n+1}$ for $K_{n+1}^{\prime}$ because it is the distance 

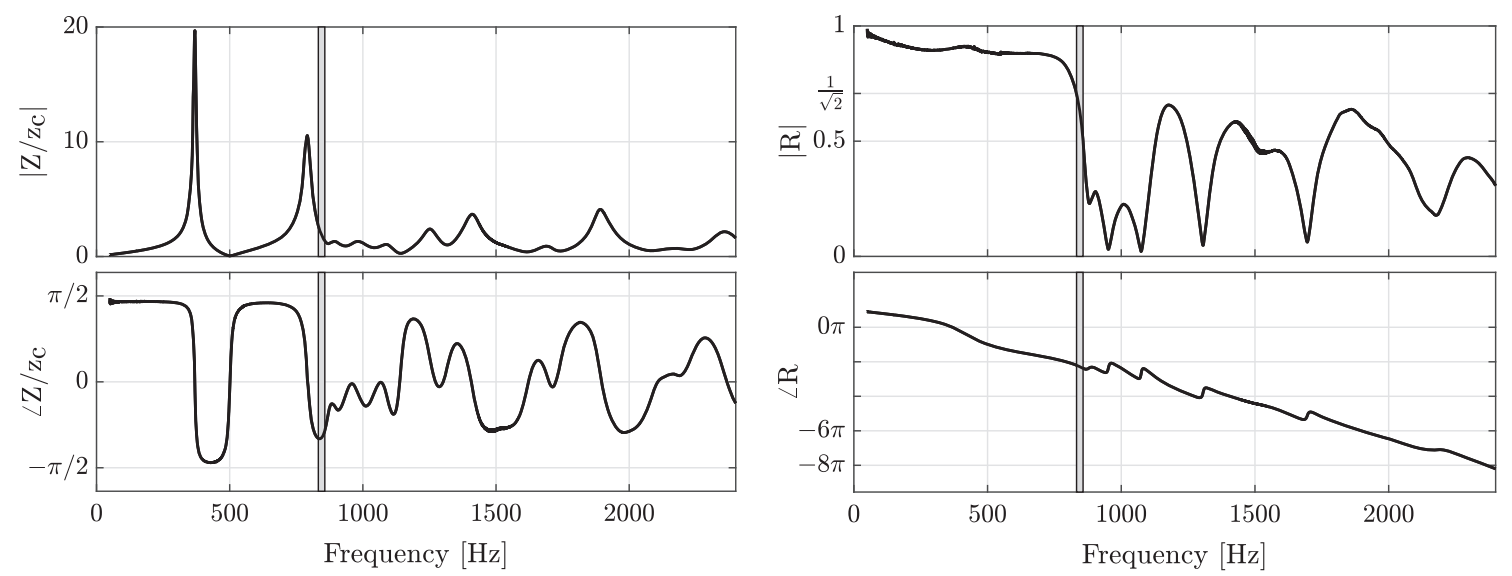

Figure 4. The input impedance (modulus and argument) and reflection coefficient (modulus and argument), measured for the high C fingering (written pitch, $311 \mathrm{~Hz}$ ) of an alto saxophone. The cutoff transition band $f_{\mathrm{t}}^{\mathrm{R}}$ in grey.
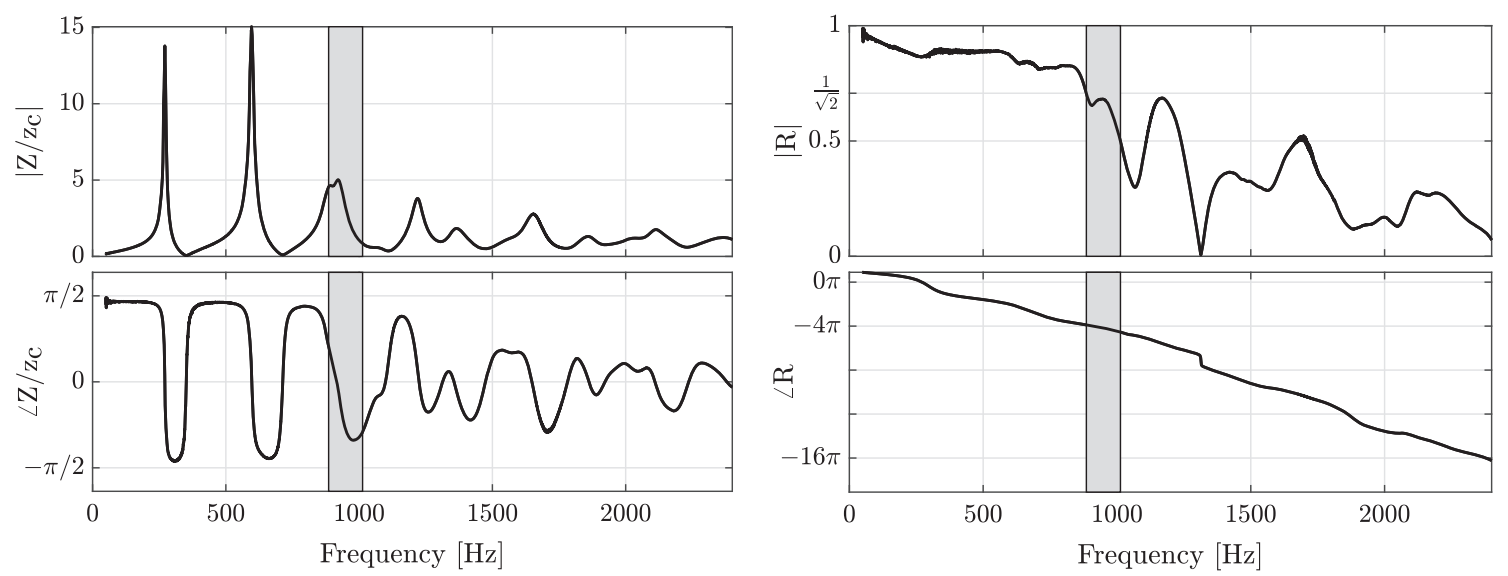

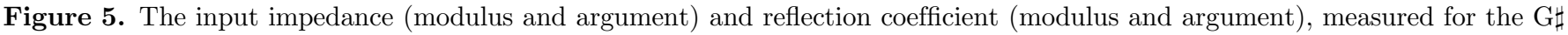
fingering (written pitch, $246 \mathrm{~Hz}$ ) of an alto saxophone. The cutoff transition band $f_{\mathrm{t}}^{\mathrm{R}}$ in grey.

between the $n$th and $(n+1)$ th holes, which is a shared quantity. Retaining two terms of the Taylor expansion yields the characteristic frequency,

$$
\begin{aligned}
f_{\mathrm{c}}^{\Pi}= & \frac{c}{2 \pi} \frac{2}{d_{n} \sqrt{3}}\left[\left(\frac{1}{3}+K_{n}+K_{n+1}^{\prime}\right) /\left[\left(\left(\frac{1}{3}\right)^{2}+\frac{2}{3}\left(K_{n}+K_{n+1}^{\prime}\right)\right.\right.\right. \\
& \left.\left.\left.+2 K_{n} K_{n+1}^{\prime}\right)^{2}-4 K_{n}^{2} K_{n+1}^{\prime 2}\right]^{1 / 2}+\mathcal{O}\left(\left(k_{\mathrm{c}}^{\mathrm{T}} \ell_{n}\right)^{3}\right)\right]
\end{aligned}
$$

If the lattice is acoustically regular then the characteristic frequency $f_{c}^{\Pi}$ will be the same for each pair of adjacent toneholes. If the lattice is not acoustically regular, as is the case for a saxophone, then the variation of $f_{\mathrm{c}}^{\Pi}$ across the lattice can be treated as a measure of the degree of acoustical irregularity of a tonehole lattice. This is different from Keefe's interpretation, in which the local cutoff frequency is defined by the characteristic frequency $f_{\mathrm{c}}^{\mathrm{\Pi}}$ of the first tonehole pair [9]. Here, all cells are considered and their variation throughout the lattice is interpreted as a deviation from perfect acoustic regularity.

Figure 7 shows the results of this theory applied to the geometry of an alto saxophone following a decreasing chromatic scale along the horizontal axis. For each fingering, the characteristic frequency $f_{\mathrm{c}}^{\Pi}$ of the first three pairs of open toneholes (when three are available, the lowest notes of scale have only one or two tonehole pairs available) are marked with circles and labeled in order with Roman numerals. The cutoff transition band $f_{\mathrm{t}}^{\mathrm{R}}$ is marked in light grey and asymmetric division (see Sect. 3.2) in dark grey.

There is considerable variation of $f_{\mathrm{c}}^{\Pi}$ from cell to cell, ranging from $500 \mathrm{~Hz}$ for cells near the end of the lattice up to $1500 \mathrm{~Hz}$ for the highest cells. This indicates that the resonator cannot be considered acoustically regular. The variation is much greater than for the clarinet, which has cutoffs that typically range from $1150 \mathrm{~Hz}$ to $1450 \mathrm{~Hz}$ [8]. A visual inspection of the input impedance of all the fingerings (not shown) confirms that the cutoff increases substantially with increasing notes on the scale. 


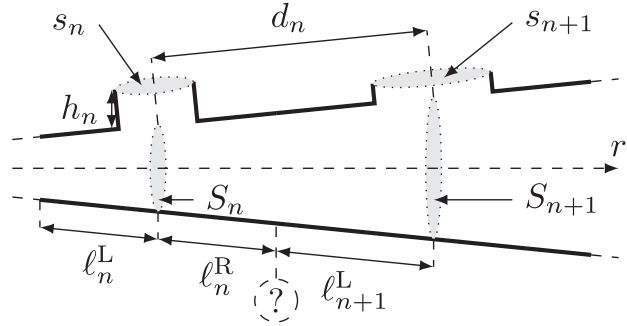

Figure 6. Schematic of a tonehole $\Pi$-cell. Interhole spacing $d_{n}=\ell_{n}^{\mathrm{R}}+\ell_{n+1}^{\mathrm{L}}$ is unambiguously defined.

The characteristic frequencies for the first three open tonehole pairs follow the same downward trend as the measured global cutoff transition band for each fingering. This implies that an empirically observed cutoff on the input impedance or reflection coefficient is largely determined by the upstream (closest to the mouthpiece) open toneholes of the lattice for a given fingering. It is worth remarking that the (approximate) cutoff frequencies of the lowest notes in the scale are due to some combination of the conicity and flare of the bell, and can not be interpreted as the result of a tonehole lattice because there are not enough open toneholes to apply the theory of wave propagation in periodic media. This ambiguity is mentioned in Section 2.4, and demonstrates again why the tonehole lattice cutoff frequency is difficult to interpret for conical resonators.

\subsection{Iterative cell division}

The second method iteratively divides a tonehole lattice into acoustically regular asymmetric T-cells, as was done for the clarinet [1]. In contrast to Section 2, the tonehole is not necessarily located at the middle of the cell. This is permissible because the important acoustic components are the acoustic mass of the tonehole and acoustic compliance of the main bore, neither of which, at low frequencies, is impacted by the location of the tonehole. The derivation follows the same steps as that of equation (12), except that in the asymmetric case the transfer matrix coefficient condition for the local cutoff frequency is $\left|A_{n}(k)+D_{n}(k)\right|=2$ with $A_{n}(k) \neq D_{n}(k)[6]$.

The $n$th asymmetric cell with lengths $\ell_{n}^{\mathrm{L}}$ and $\ell_{n}^{\mathrm{R}}$ to the left and right of the tonehole has a local cutoff,

$$
f_{\mathrm{c}}^{\mathrm{T}}=\frac{c}{2 \pi\left(\ell_{n}^{\mathrm{L}}+\ell_{n}^{\mathrm{R}}\right)}\left[\frac{1}{\sqrt{\left(h_{n} /\left(\ell_{n}^{\mathrm{L}}+\ell_{n}^{\mathrm{R}}\right)\right)\left(S_{n} / s_{n}\right)}}+\mathcal{O}(k \ell)\right] .
$$

As in equation (12), this is an approximate definition due to the number of terms retained in the Taylor expansion in $k \ell$ of the cotangent function.

A lattice can then be divided using iteration (see Fig. 8) by choosing the initial length $\ell_{1}^{\mathrm{L}}$ for the first cell and

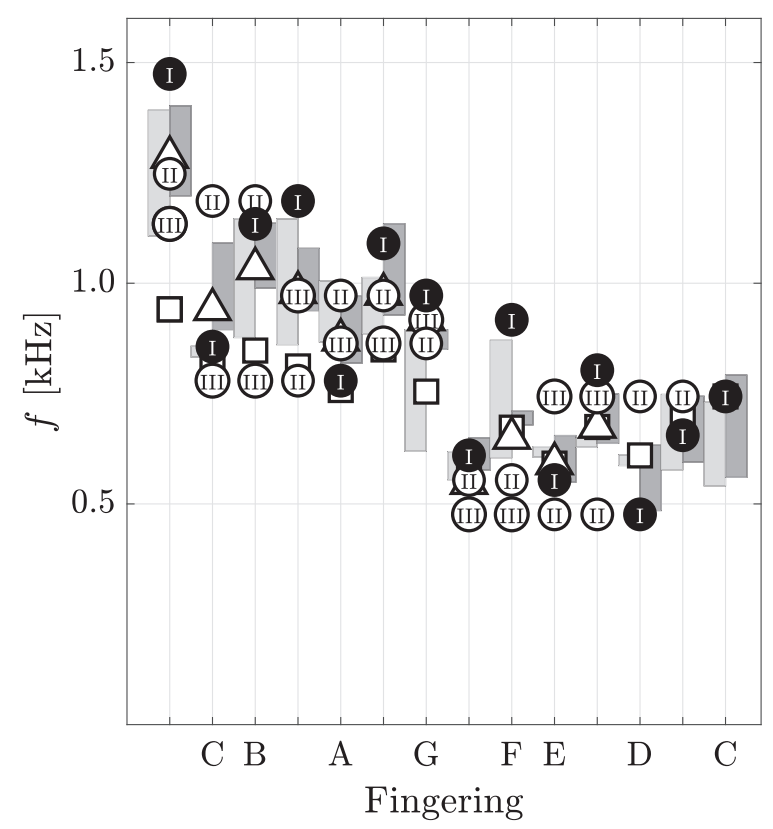

Figure 7. Estimation of the cutoff frequency for each fingering down to the low $C$ (written, $156 \mathrm{~Hz}$ ) of an alto saxophone. Data markers correspond to: the first three $f_{\mathrm{c}}^{\Pi}$ : (I III III); average of first three $f_{\mathrm{c}}^{\Pi}: \Delta$; average of all $f_{\mathrm{c}}^{\Pi}: \square$ asymmetric T-cells (see Sect. 3.2): $\quad$; transition band $f_{\mathrm{t}}^{\mathrm{R}}$ : $\square$.

imposing a local cutoff frequency $f_{\mathrm{c}}^{\mathrm{T}}$. Because the tonehole dimensions are given, only $\ell_{1}^{\mathrm{R}}$ can be varied to achieve the local cutoff frequency. The length of the right portion of the first cell $\ell_{1}^{\mathrm{R}}$, along with the distance to the next hole $d_{1}$, determines the left section of the next hole $\ell_{2}^{\mathrm{L}}$, and so forth for the entire lattice, see Figure 6 . A division of the lattice is attempted for a wide range of initial lengths and eigenfrequencies: $\ell_{1}^{\mathrm{L}} \in\left[0, d_{1}\right] \mathrm{m}$ and $f_{\mathrm{c}}^{\mathrm{T}} \in[0.1,3] \mathrm{kHz}$. Although it is mathematically possible for $\ell_{n}^{\mathrm{L}}$ and $\ell_{n}^{\mathrm{R}}$ to be complex, a lattice is only considered successfully divided if all $\ell_{n}^{\mathrm{L}}$ and $\ell_{n}^{\mathrm{R}}$ are real and positive, ensuring a physically realizable lattice. A successful division means that each cell of the lattice has the same local cutoff $f_{c}^{\mathrm{T}}$, so the lattice is acoustically regular with a global cutoff $f_{c}^{\mathrm{G}}$ at this frequency. Because the constituent cells are allowed to be asymmetric, a single lattice can have a continuum of successful divisions, and the span of this continuum is interpreted as the breadth of admissible frequencies for which the resonator can be considered acoustically regular.

The asymmetric division algorithm is applied to two acoustically regular lattices designed following the criteria in Section 2.4 with global cutoff frequencies at 0.75 and $1.0 \mathrm{kHz}$. The dark grey region in Figure 9 depicts the continuum of successful asymmetric divisions of the two lattices. Additionally, the tonehole pair characteristic frequencies $f_{\mathrm{c}}^{\Pi}$, marked as a dashed line, are all equal because the lattices are acoustically regular so $f_{\mathrm{c}}^{\mathrm{T}}=f_{\mathrm{c}}^{\Pi \mathrm{T}}$ for all cells. The cutoff transition band $f_{\mathrm{t}}^{\mathrm{R}}$ is shown in light grey. These plots show the coherence between symmetric and asymmetric cells, tonehole pair characteristic frequencies 


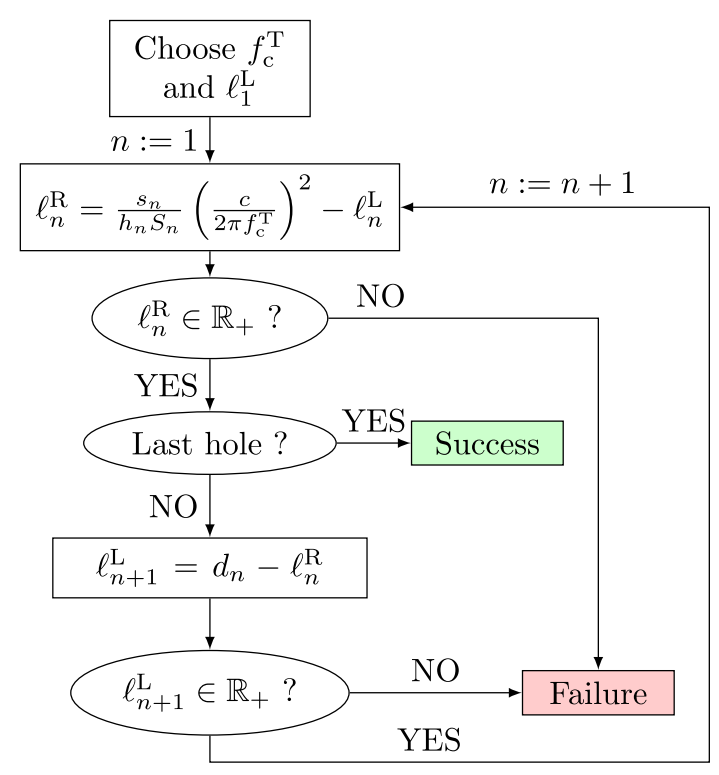

Figure 8. A flowchart of the asymmetric division algorithm described in Section 3.2 used to divide the acoustically regular lattices shown in Figure 9 and alto saxophone in Figure 10.

$f_{\mathrm{c}}^{\Pi}$, and the reflection coefficient cutoff transition band $f_{\mathrm{t}}^{\mathrm{R}}$ when the resonator is acoustically regular.

The asymmetric division algorithm is applied to the geometry of a Buffet Crampon alto saxophone. Successful division of the lattice is only possible for select fingerings, all in the bottom range of the instrument. The reason for this is that several of the lowest toneholes have highly irregular spacing, and the asymmetric division fails to find a division of these cells for the eigenfrequencies possible for the upstream cells. This implies that the method of asymmetric division may be better suited for resonators that are highly acoustically regular, and application to the saxophone must be treated carefully. However, if the lowest three holes are excluded from the algorithm, division is possible for most notes. Division across all fingerings is possible when only the first 5 tonehole pairs (6 toneholes) of each fingering is included in the algorithm. The division for fingerings $\mathrm{C}$ and $\mathrm{G} \sharp$ are shown in Figure 10, and their measured input impedance and reflection functions are provided in Figures 4 and 5 , respectively. As before, the cutoff transition band $f_{\mathrm{t}}^{\mathrm{R}}$ is shown in light grey. The characteristic frequencies of the first three cells are marked by circles (black, grey, white, respectively), the average of the three by a triangle and the average of the whole lattice by a square.

Figures 4 and 5, corresponding to the high $\mathrm{C}$ and $\mathrm{G} \sharp$ fingerings, appear to be quite different. However, the results of the asymmetric division are quite similar: the $\mathrm{C}$ fingering division ranges between 940 and $1120 \mathrm{~Hz}$, and the $\mathrm{G} \sharp$ fingerings between 930 and $1140 \mathrm{~Hz}$. The difference in the range of initial lengths $\ell_{1}^{\mathrm{L}}$ is not important because it is simply an arbitrary length of the resonator upstream from

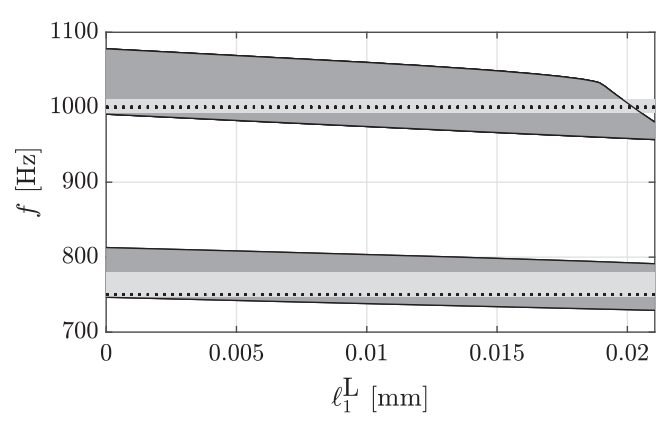

Figure 9. Cutoff frequencies found using asymmetric division (dark grey region) of acoustically regular conical lattices with global cutoff frequencies at $750 \mathrm{~Hz}$ (bottom) and $1000 \mathrm{~Hz}$ (top) for different initial cell length $\ell_{1}^{\mathrm{L}}$. The characteristic frequencies $f_{\mathrm{c}}^{\Pi}=f_{\mathrm{c}}^{\mathrm{T}}$ are marked by a dashed line, and the cutoff transition band $f_{\mathrm{t}}^{\mathrm{R}}$ by light shaded grey.

the first tonehole used to initiate the algorithm. As long as the algorithm arrives at a successful solution, the initialization does not matter because the degree of acoustic regularity is related to the span of possible global cutoff frequencies, and not the initial length $\ell_{1}^{\mathrm{L}}$. Therefore, although the continuum of successful divisions appear quite different for the two fingerings, the span of admissible global cutoff frequencies are quite similar.

The cutoff transition band $f_{\mathrm{t}}^{\mathrm{R}}$ is very narrow for the high $\mathrm{C}$ and relatively broad for the $\mathrm{G} \sharp$. This is also evident in Figures 4, 5, and 7. Compared with other fingerings, the severity of the drop in the reflection function for the $\mathrm{C}$ fingering is unusually abrupt, and the width of the cutoff transition band varies considerably between fingerings.

\subsection{Observations}

Each type of analysis $\left(f_{\mathrm{t}}^{\mathrm{R}}, f_{\mathrm{c}}^{\Pi}\right.$, and asymmetric division of $f_{\mathrm{c}}^{\mathrm{T}}$ ) applied to the saxophone has advantages and disadvantages, and provides a different nuance to wave propagation in conical lattices. All three methods demonstrate an increasing cutoff for increasing notes in the first register as seen in Figure 7, which is consistent with observations of the input impedance of each note. This implies that all of these methods can be used to analyze the cutoff frequency of an alto saxophone, and the choice depends on the specific requirements of the study.

The cutoff frequency transition band and $\Pi$-cell calculations are likely the easiest methods to analyze a lattice, requiring either an input impedance measurement or the lattice geometry. These avoid complications that can arise using asymmetric division, such as the choice of how many cells to include when attempting to divide a highly irregular lattice. The transition band is particularly appealing in the case of real woodwind instruments because it directly measures a cutoff behavior of the resonator, with no assumptions about acoustic regularity. However, it is not clear how well this method would work for other instruments, 

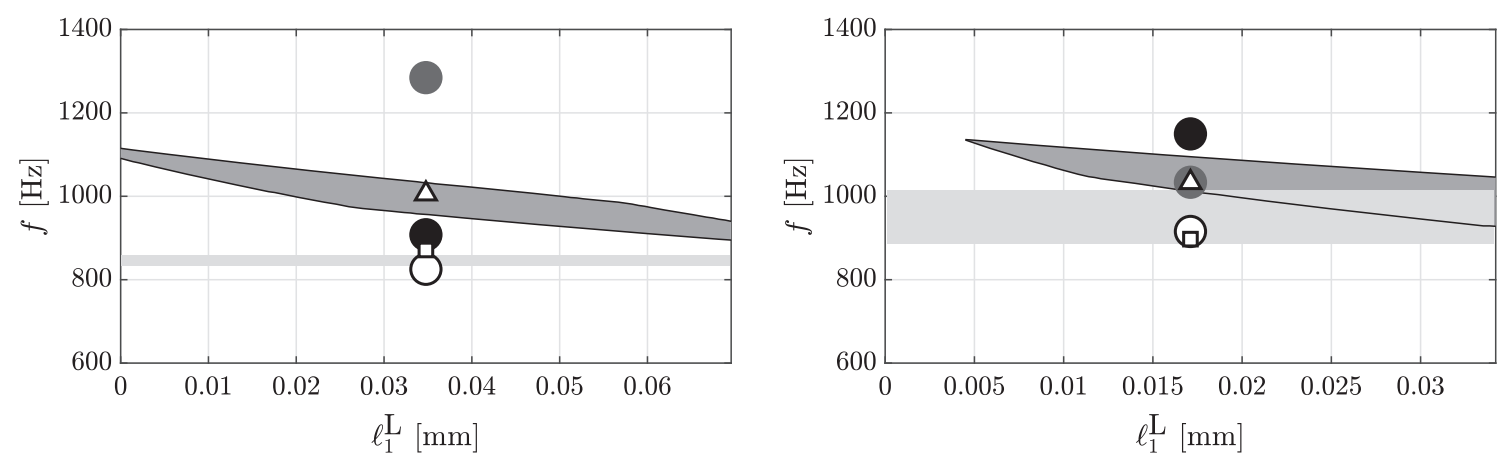

Figure 10. Comparison of three analysis methods. Successful asymmetric division (dark grey region) of an alto saxophone high C on left (written pitch, $311 \mathrm{~Hz}$ ) and $\mathrm{G} \sharp$ on right (written pitch, $246 \mathrm{~Hz}$ ) fingerings for different initial cell length $\ell_{1}^{\mathrm{L}}$. The first three tonehole pair cell characteristic frequencies $f_{c}^{\Pi}$ are denoted $\bigcirc$. The average of the first three $\Pi$-cells and of the entire lattice are provided by $\Delta$ and $\square$. The cutoff transition band $f_{\mathrm{t}}^{\mathrm{R}}$ is shown in light grey.

and it is possible that the thresholds would need to be adjusted for different instrument families.

\section{Conclusion}

This article provides some insights into why the cutoff frequency due to the toneholes of the saxophone is understudied compared with other instruments. The impossibility to define symmetric cells in a conical geometry is a difficulty that hinders all attempts of analytical study. An acoustic regularity can be defined to overcome the inherent geometric irregularity of a conical tonehole lattice, which can be used to design lattices to have a desired local cutoff. Even then, intrinsic acoustical characteristics of cones lead to more diffuse cutoff behavior than with cylinders. For real instruments, the influence of the bell, particularly for the lowest notes on the instrument, may contribute to the diffuse cutoff. Further work on this topic could include an investigation into the parameters that influence the efficiency of the cutoff, particularly for non-cylindrical resonators.

It is generally assumed that the cutoff remains fairly constant for different fingerings of a given instrument. Benade provides figures showing this phenomena for both cylindrical and conical instruments, the oboe, bassoon, and clarinet [3]. As seen here, the cutoff of the saxophone varies considerably across the range of one octave. Many notes, particularly in the higher range of the register starting around $F$ (written pitch, $208 \mathrm{~Hz}$ ), have only two impedance peaks to collaborate with the reed. Below $F$ some notes have three or four strong impedance peaks, while the lowest notes with only one or two holes open have many more undisturbed impedance peaks. It would be interesting to study this in terms of perceived homogeneity of the timbre in the radiated sound field for different notes on the instrument. In link with sound production features, future studies could evaluate the role of the instrument maker in consciously setting the cutoff properties of the tonehole lattice, notably in compromise with the other design parameters.

\section{Acknowledgments}

The authors appreciate insightful conversations and suggestions from Douglas Keefe. Thanks to Michael Jousserand and Buffet Crampon for providing the geometric measurements of the alto saxophone, as well as Patrick Sanchez and the Service Études et Réalisations Mécaniques for their help in preparing these measurements. This work has been partly supported by the French Agence Nationale de la Recherche (ANR16LCV2-0007-01 Liamfi project).

\section{References}

1. E. Moers, J. Kergomard: On the cutoff frequency of clarinetlike instruments. Geometrical versus acoustical regularity". Acta Acustica United With Acustica 97 (2011) 984-996.

2. J. Wolfe. http://newt.phys.unsw.edu.au/music/. Website about musical acoustics. Accessed 2020.

3. A.H. Benade: Fundamentals of Musical Acoustics. Oxford University Press, London, 1976.

4. J. Wolfe, J. Smith: Cutoff frequencies and cross fingerings in baroque, classical, and modernutes. Journal of the Acoustical Society of America 114, 4 (2003) 2263-2272.

5. E. Petersen, P. Guillemain, J. Kergomard, T. Colinot: The effect of the cutoff frequency on the sound production of a clarinet-like in instrument. Journal of the Acoustical Society of America 145, 6 (2019) 3784-3794.

6. L. Brillouin, M. Parodi: Wave propagation in periodic structures. Mc Graw Hill, New York, NY, USA, 1946.

7. A.H. Benade, S.J. Lutgen: The saxophone spectrum. Journal of the Acoustical Society of America 83 (1988) 1900-1907.

8. A. Chaigne, J. Kergomard: Acoustics of Musical Instruments. Springer-Verlag, New York, 2016. (English Translation).

9. D.H. Keefe: Woodwind tone hole acoustics and the spectrum transformation function. PhD Thesis. Department of Physics, Case Western Reserve University, 1981.

10. D.H. Keefe: Woodwind air column models. Journal of the Acoustical Society of America 88 (1990) 35-51.

11. J. Kergomard: Internal field and external field of wind instruments, PhD thesis, Université Paris 6 (in French), 1981.

12. A. Lefebvre, G.P. Scavone, J. Kergomard: External tonehole interactions in wood-wind instruments. Acta Acustica United With Acustica 99 (2013) 975-985. 


\section{Appendix}

Appendix provides the geometries for the two acoustically regular lattices used in this article. Both are used to test the asymmetric iterative division algorithm, Figure 9, described in Section 3.2. The simulated input impedance and reflection coefficient of the first resonator, shown in Figure 1, demonstrates the methodology of designing a conical resonator with an acoustically regular lattice presented in Section 2. The equivalent figure for the second resonator is not included to omit redundancy.

Both resonators have an apex angle $\theta=0.028 \mathrm{rad}$ and in input radius $a_{\text {in }}=6.25 \mathrm{~mm}$, corresponding to the geometry of the alto saxophone analyzed in Section 3. The equations governing an acoustically regular lattice provide a wide range of options for the geometry of the toneholes. The geometries presented here attempt to follow general trends of an alto saxophone, although they are necessarily different because the saxophone is not acoustically regular.
Table A1. Dimensions of two acoustically regular resonators with different first impedance peak and theoretical global cutoff frequencies. Both resonators have an apex angle $\theta=0.028 \mathrm{rad}$ and input radius $a_{\text {in }}=6.25 \mathrm{~mm}$. The distance from the input to the $n$th hole is denoted $w_{n}=r_{n}-x_{1}$, where $x_{1}$ is determined from the the apex angle and input radius.

\begin{tabular}{lcccc}
\hline \multicolumn{4}{l}{ Resonator $1: f_{1}=177 \mathrm{~Hz}, f_{\mathrm{c}}^{\mathrm{G}}=750 \mathrm{~Hz}$} \\
Hole $n$ & $w_{n}(\mathrm{~mm})$ & $a_{n}(\mathrm{~mm})$ & $b_{n}(\mathrm{~mm})$ & $h_{n}(\mathrm{~mm})$ \\
\hline 1 & 712.9 & 26.2 & 10.5 & 13.2 \\
2 & 744.5 & 27.1 & 12.3 & 15.4 \\
3 & 779.4 & 28.1 & 14.4 & 17.0 \\
4 & 818.0 & 29.2 & 16.5 & 17.9 \\
5 & 860.6 & 30.4 & 18.9 & 18.0 \\
6 & 907.7 & 31.7 & 21.5 & 17.4 \\
7 & 959.7 & 33.1 & 24.3 & 15.8 \\
8 & 1017.3 & 34.7 & 27.4 & 13.2 \\
9 & 1080.9 & 36.5 & 30.8 & 9.6 \\
10 & 1151.2 & 38.5 & 34.6 & 4.8 \\
end & 1188.0 & 395.2 & - & - \\
\hline Resonator & $2:$ & $f_{1}=304 \mathrm{~Hz}, f_{\mathrm{c}}^{\mathrm{G}}=1000 \mathrm{~Hz}$ & & \\
\hline 1 & 355.7 & 15.6 & 6.2 & 14.9 \\
2 & 376.7 & 16.2 & 7.2 & 16.3 \\
3 & 400.0 & 16.8 & 8.2 & 17.2 \\
4 & 425.7 & 17.5 & 9.3 & 17.8 \\
5 & 454.1 & 18.3 & 10.6 & 17.8 \\
6 & 485.5 & 19.1 & 11.9 & 17.4 \\
7 & 520.3 & 20.1 & 13.4 & 16.4 \\
8 & 558.6 & 21.1 & 15.0 & 14.8 \\
9 & 601.0 & 22.2 & 16.8 & 12.7 \\
10 & 647.9 & 23.5 & 18.8 & 9.9 \\
end & 672.5 & 24.2 & - & - \\
\hline & & & &
\end{tabular}

Cite this article as: Petersen E, Colinot T, Kergomard J \& Guillemain P. 2020. On the tonehole lattice cutoff frequency of conical resonators: applications to the saxophone. Acta Acustica, 4, 13. 